\title{
Using mobile augmented reality to enhancing students' conceptual understanding of physically-based rendering in 3D animation
}

\author{
Tiantada Hiranyachattada1,* and Kampanat Kusirirat ${ }^{2}$ \\ 1,* Department of Animation and Digital Media, Faculty of Science and Technology, Bansomdejchaopraya Rajabhat University, \\ Bangkok, 10600, Thailand. \\ 2 Department of Animation and Digital Media, Faculty of Science and Technology, Bansomdejchaopraya Rajabhat University, \\ Bangkok, 10600, Thailand. \\ For correspondence: bmafueng@hotmail.com
}

\begin{abstract}
:
Physically-based rendering (PBR) concept is widely use nowadays in 3D rendering works, this concepts interested in the ray of light for describes the interaction of light and materials. Understanding the principles of PBR can be easier to adjust shader parameters to be realistic, react correctly to changes in lighting condition and also giving the same results even in different 3D rendering software. In Shading lighting and rendering (SLR) subject which PBR concepts become important concept instead the 'old style rendering' it was found that students are unable to clearly understand concepts of PBR. According to the problem, in this research bringing mobile augmented reality (AR) to be a media for demonstrate PBR concepts for students. The sample were 35 students from Department of Animation and Digital Media, Bansomdejchaopraya Rajabhat University. The results found that students understand the PBR concepts and can adjust the PBR shader paremeters to be realistic assess from students pre-test, post-test scores and students homework. The mobile AR application media were usable for students and suitable to be a learning media in 3D animation assess form students' response.
\end{abstract}

Keywords: shader, 3D animation, PBR, augmented reality

\section{Introduction}

3D rendering works has been being developed rapidly nowadays from the advances in physics, mathematics and computer science as can be seen from 3D animation and 3D games. Physically-based rendering (PBR) is a method of shading and rendering that interested in the ray model of light as it describes the interaction of light and surface matter to be similar in virtual worlds. The key characteristics of PBR is energy conservation rule which means reflected ray is never brighter than the value it had when it first affect the surface (Substance Academy, 2019). Physically-based rendering (PBR) concepts are widely use nowadays in 3D rendering works togather with shader which is a computer program that used for calculate shading such as the production of light, darkness, and colors (Claudia Doppioslash, 2019).

To adjusting the shader parameters in 3D animation works can be divide into 2 types: 'old style rendering' which is parameters can be flexible and freely to adjust that can give unrealistic results depend on shader artist and 'PBR' concepts rendering which is a newer concepts that focus on the physics to light. Understanding the PBR concepts can be easier to adjust shader parameters to be realistic same as in the virtual world, react correctly to changes in lighting condition and also giving the same results even in different $3 \mathrm{D}$ rendering software.

At present, learning 3D animation this concept is not used widely use especially in Shading lighting and rendering (SLR) subject, was one of the important subject which have focused on shader, lighting, 
and rendering which PBR concepts become important concept instead the 'old style rendering'. It was found that students are unable to clearly understand PBR concepts and misunderstanding each parameter values.

As the problem mentioned, to making students understand PBR concepts in this research bringing augmented reality (AR) technology via moblie phone which believe that the use of AR technology media can improve aspects of learning also physical phenomenon such as light and can be apply with AR technology in many form such as animation or virtual labatory (Jennifer L et al., 2015) to demonstrate PBR concepts in 3D animation for students in SLR subject and provide learinging experience and knowladge to be understood by the students (M A Muchtar et al., 2016) for 3D animation works.

\section{Mobile AR application media design and creation}

After identifying the learning problems in SLR subject, we will creating moblie AR application to be a learning media for students, the moblie AR application was developed by using software development life cycle whice are software specification, software design, software implementation and testing (Langer, Arthur M., 2018) with Unity (Unity Technologies, 2018 ; Rod Lopez, 2014) and Vuforia (Vuforia ${ }^{\mathrm{TM}}$, a PTC Technology, 2018). The mobile AR application begins with the virtual world object detection of the examples objects after the object in the real world has been detected the moblie AR application will display the 3D model of the objects and its PBR parameters value. The mobile AR application system creation as shown in figure 1.

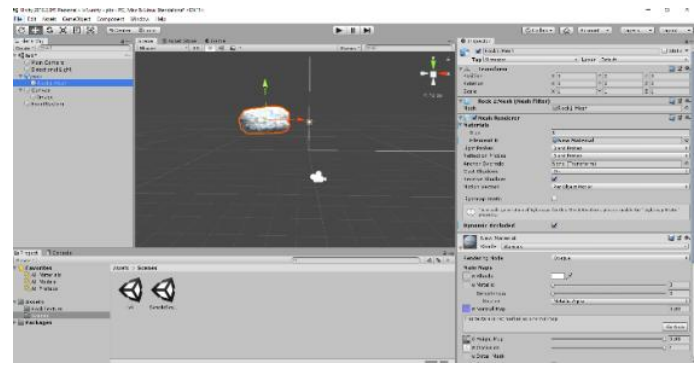

Figure 1. Crating mobile AR application with Unity

\section{Method and Classroom demonstration}

The method used in this research was experiment method with one-group pretest posttest design. In methodology, the pretest was first assinged to the subject to evaluate student's knowledge in shader adjustment that students had lerned from pre-ruquisit subject. After finish the pretest, the demonstration begin with giving the real object then students will use the mobile AR application to scan the example objects with their mobile phone until the mobile AR application display the 3D model of the real object in PBR concepts and PBR key parameters. After finished demonstration, the post-test was administered to measure the effect of learning AR media according to the examples given also in each weeks of the study the lecturer will give students homework in order to validate the understanding and continuously implement the knowledge. The learning framework of PBR concepts as shown in figure 2 and the mobile AR application as shown in figure 3.

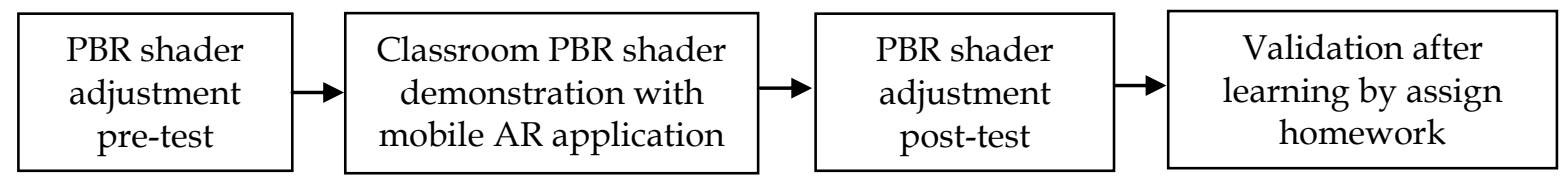

Figure 2. Learning framework 


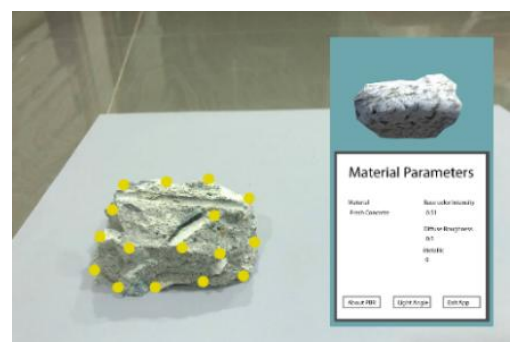

Figure 3. mobile AR application interface

\section{Results}

The 35 students sample were from Department of Animation and Digital Media, Bansomdejchaopraya Rajabhat University, Bangkok, Thailand, in the 1st semester of academic year 2019 selecting by purposive sampling methods. For the pre-test, the students' score in average were lower than $50 \%$ of full scores which mean students cannot adjust the shader parameters in PBR concepts as can be seen from material that look unrealistic. The student's pre-test and post-test scores as shown in table 1.

Table 1. Statistical data of students' conceptual understanding score in PBR

\begin{tabular}{lccc}
\hline \multirow{2}{*}{ objects } & & Pre-test & Post-test \\
\cline { 3 - 4 } \cline { 3 - 4 } & $\mathrm{N}$ & Mean (Std.Dev) & Mean (Std.Dev) \\
\hline Concrete (5 points) & 35 & $2.14(0.85)$ & $4.43(0.50)$ \\
Aluminium (5 points) & 35 & $2.11(0.80)$ & $4.57(0.50)$ \\
Plastic (5 points) & 35 & $2.17(0.66)$ & $4.51(0.51)$ \\
\hline
\end{tabular}

For the post-test, students' scores were higher than the pre-test scores. The results presented that the scores were increasing more than $50 \%$ from 2.14 to $4.43,2.11$ to 4.57 and 2.17 to 4.51 . Moreover, students can adjust the shader parameters in PBR concepts [8] correctly and understand the PBR concept clearly from visualization though 3D objects also can tell the difference between 'old style rendering' and PBR concepts rendering. The students' pre-test and post-test result examples as shown in figure 4 .
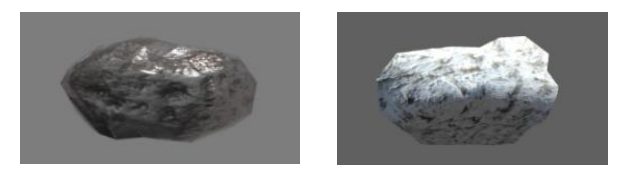

(a)

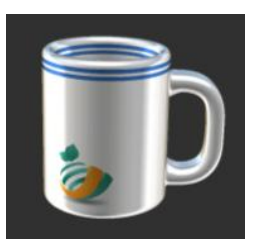

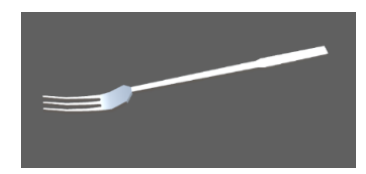

(b)

(c)

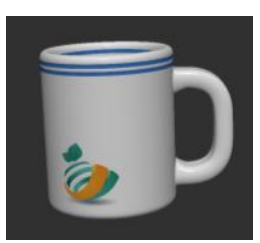

Figure 4. Like-for-like comparison between non-PBR and PBR from student's pre-test and post-test

(a) concrete (b) aluminium and (c) plastic

For validating students' knowledge, in each week of the SLR subject, the lecturer will assign homework for students which were 3D animation scene such as exterior scene and interior scene also 
grade student's homework by rubrics score. In table 2 shown the students homework scores and in figure 5 shown the students 3D animation scene homework examples.

Table 2. Student's homework scores

\begin{tabular}{|c|c|c|}
\hline Week-Topics & $\mathbf{N}$ & Mean (Std.Dev) \\
\hline 1 - Exterior small scene (10 points) & 35 & $9.11(0.80)$ \\
\hline 2 - Exterior scene (10 points) & 35 & $8.71(0.75)$ \\
\hline 3 - Interior scene (10 points) & 35 & $8.40(1.14)$ \\
\hline 4 - Interior scene (10 points) & 35 & $8.37(1.14)$ \\
\hline Average scor & & $8.64(0.95)$ \\
\hline
\end{tabular}

The student's homework scores shown that student's average scores in each week were higher than $80 \%$ and students can apply shader parameters in PBR concepts with different 3D animation scenes.

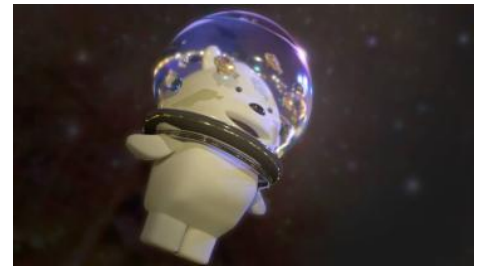

(a)

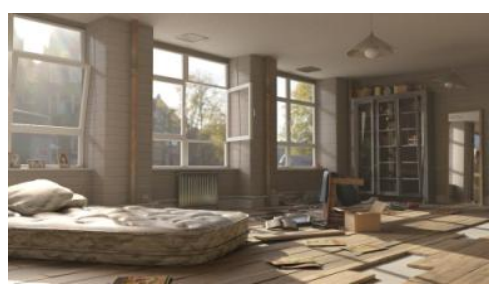

$(\mathrm{c})^{*}$

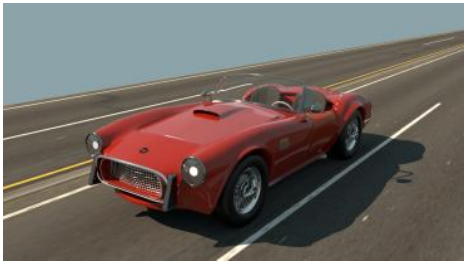

$(b)^{*}$

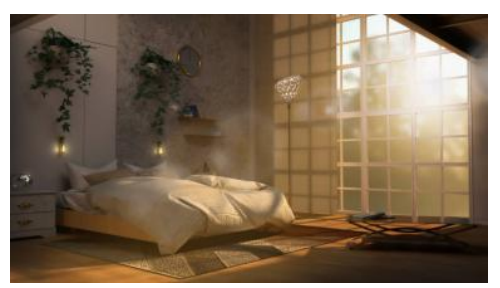

$(\mathrm{d})^{*}$

Figure 5. Student's homework examples in (a) and (b) exterior scene (TurboSquid, 2018),

(c) interior scene (Solid angle, 2018) and (d) interior scene (Mographplus, 2018)

After using mobile AR application to demonstrate PBR concepts in 3D animation, 35 students give their responses from questionnaires (Irwansyah, F et al., 2018) towards the use of mobile AR application. The results as shown in table 3 .

Table 3. Students' response towards the use of mobile AR application by questionnaire

\begin{tabular}{lcc}
\hline \multicolumn{1}{c}{ Topics } & \multicolumn{2}{c}{ Mean (Std.Dev) } \\
\hline Learning outcome & & \\
Learning objective & $4.44(0.50)$ & Good \\
Increasing students learning motivation & $4.56(0.50)$ & Very good \\
Suitable to be applied in the process of learning PBR & $4.38(0.55)$ & Good \\
AR Application & & \\
AR application interface & $4.34(0.48)$ & Good \\
The usage of AR application & $4.47(0.51)$ & Good \\
\hline & & Good \\
\hline
\end{tabular}

The students' response shown that the mobile AR application can meet the learning objectives and usable for students is this subject. The increasing students learning motivation topic had the highest scores at 4.56 which mean AR media was interesting and can be attracted for students and AR 
application interface had the lowest score at 4.34 which mean the application interface must be improve to be more human friendly and better design.

\section{Conclusion and Discussion}

Based on the demonstration and the results, the conclusions were: mobile AR application can make students understand the concept of PBR though 3D model which the most important features of AR could make students can rotate the 3D model freely, making them easier to see the 3D model from various perspectives. Students have better observation and analyzing skills, can compare the PBR model parameters with the real object, so students can learn and tell the differences and can adjust the shader parameters for 3D animation. Hence, this media gets positive responses from students and suitable to be applied in the process of learning PBR for 3D animation or other 3D animation rendering concepts in the future.

\section{Acknowledgements}

This research was supported by Research and Development Institute and Faculty of science and technology, Bansomdejchaopraya Rajabhat University, Bangkok, Thailand.

\section{References}

Arnold Renderer, (2018), Autodesk I Arnold Renderer https://www.arnoldrenderer.com, (accessed October 2018)

Chao, J., Chiu, J., DeJaegher, C., \& Pan, E. (2015). Sensor-Augmented Virtual Labs: Using Physical Interactions with Science Simulations to Promote Understanding of Gas Behavior. Journal Of Science Education And Technology, 25(1), 16-33.

Doppioslash, C, (2019), What is a Shader?https://www.shadercat.com/what-is-a-shader/, (accessed December 2018)

Epic Games, (2018). Physically Based Materials https://docs.unrealengine.com/enus/Engine/Rendering/Materials/PhysicallyBased (accessed April 2018)

Irwansyah, F., Yusuf, Y., Farida, I., \& Ramdhani, M. (2018). Augmented Reality (AR) Technology on The Android Operating System in Chemistry Learning. IOP Conference Series: Materials Science And Engineering, 288, 012-068.

LANGER, A, (2018). GUIDE TO SOFTWARE DEVELOPMENT. (pp. 1-200). [Place of publication not identified]: SPRINGER.

Lopez, R, (2019). Physically Based Shading in Unity 5: A Primer - Unity Blog, https://blogs.unity3d.com/2014/10/29/physicallybased-shading-in-unity-5-a-primer, (accessed October 2018)

Muchtar, M., Syahputra, M., Syahputra, N., Ashrafia, S., \& Rahmat, R, (2017). Augmented Reality for Searching Potential Assets in Medan using GPS based Tracking. Journal Of Physics: Conference Series, 801, 1-6.

Solid Angle, (2018). Comprehensive Introduction to Arnold 5 for Maya, http://mographplus.com/product/comprehensiveintroduction-to-arnold-5-for-maya, (accessed September 2018)

Substance Academy, (2019). The PBR Guide - Part 1 on Substance Academy, https://academy.allegorithmic.com/courses/thepbr-guide-part-1 (accessed March 2018)

TurboSquid, (2018), Shelby 3D Models for Download https://www.turbosquid.com/3d-model/shelby (accessed October 2018)

Unity Technologies. (2018). Getting Started with Vuforia Engine in Unit https://library.vuforia.com/articles/Training/gettingstarted-with-vuforia-in-unity.html, (accessed March 2018)

Vuforia $^{\mathrm{TM}}$, a PTC Technology, (2018), Vuforia Developer Portal https://developer.vuforia.com/ (accessed October 2018) 